\title{
Sex Differences in Neuropsychological Functioning are Domain-Specific in Adolescent and Young Adult Regular Cannabis Users
}

\author{
George Savulich ${ }^{1}$ (1) , Natali Rychik², Erin Lamberth ${ }^{2}$, Maya Hareli ${ }^{3}$ (1) , A. Eden Evins ${ }^{2,4}$, Barbara J. Sahakian ${ }^{1}$ and \\ Randi M. Schuster ${ }^{2,4, *}$ \\ ${ }^{1}$ Department of Psychiatry and Behavioral and Clinical Neuroscience Institute, University of Cambridge, School of Clinical Medicine, Cambridge, UK \\ ${ }^{2}$ Center for Addiction Medicine, Department of Psychiatry, Massachusetts General Hospital (MGH), Boston, MA, USA \\ ${ }^{3}$ Department of Psychology, Loyola University Chicago, Chicago, IL, USA \\ ${ }^{4}$ Harvard Medical School, Boston, MA, USA
}

(Received June 3, 2020; Final Revision November 30, 2020; AcCePted December 18, 2020)

\begin{abstract}
Objective: Adolescence into young adulthood represents a sensitive period in which brain development significantly diverges by sex. Regular cannabis use by young people is associated with neuropsychological vulnerabilities, but the potential impact of sex on these relationships is unclear. Method: In a cross-sectional study, we examined sex differences in multi-domain neuropsychological functioning using the Cambridge Neuropsychological Test Automated Battery (CANTAB) and tested whether sex moderated the relationship between cognitive performance and age of initiation, frequency of cannabis use, amount of cannabis use, and withdrawal symptoms in at least weekly adolescent and young adult cannabis users $(n=171$; aged 13-25 years; $46.2 \%$ female). Results: Male cannabis users had poorer visual recognition memory and female cannabis users showed worse attention and executive functions, with medium to large effect sizes. These sex effects persisted, when controlling for age, IQ, amount of alcohol and nicotine use, mood and anxiety symptoms, emotional stability and impulsive behavior. Earlier age of initiated use and more use were associated with worse attentional functions in females, but not males. More use was more strongly associated with worse episodic memory in males than in females. More use was associated with poorer learning in males only.

Conclusions: Domain-specific patterns of neuropsychological performance were found by sex, such that males showed poorer visual memory and females showed worse performance on measures of attention (sustained visual, multitasking) and executive functioning (spatial planning/working memory subdomains). Larger studies including healthy controls are needed to determine if the observed sex differences are more exaggerated relative to non-users.
\end{abstract}

Keywords: Cannabis, marijuana, sex, neurodevelopment, neuropsychology, adolescence

\section{INTRODUCTION}

Cannabis is one of the most widely used substances in the world, and is one of the most commonly used substances among adolescents and young adults in high-income countries (Hall \& Degenhardt, 2009). In the United States, $12.5 \%$ of adolescents aged 12 to 17 years and $34.8 \%$ of young adults aged 18 to 25 years used cannabis in the past year (Substance Abuse and Mental Health Services Administration, 2019), with around 5\% of high school students reporting daily use (Johnston et al., 2020). The continued legalization and commercialization of cannabis for medicinal

*Correspondence and reprint requests to: Randi M. Schuster, Center for Addiction Medicine, Massachusetts General Hospital, 101 Merrimac Street, Suite 320, Boston, MA 02114, USA. E-mail: RSCHUSTER@mgh.harvard. edu and recreational purposes has resulted in decreased perceptions of harm and increased acceptability and availability even among youth (Cohen, 2010). In heavy adult users, poor cognitive performance has been reported in multiple domains including attention, executive functions, psychomotor speed, episodic memory, and verbal fluency (e.g. Lisdahl \& Tapert, 2012), with accumulating evidence indicating sex differences (Crane, Schuster, Fusar-Poli, \& Gonzalez, 2013; Lisdahl \& Price, 2012; King et al., 2011; Clark, Roiser, Robbins, \& Sahakian, 2009; Pope, Jacobs, Mialet, Yurgelun-Todd, \& Gruber, 1997). However, surprisingly few studies have examined how chronic cannabis use may differentially impact neuropsychological functioning in male and female cannabis users in adolescence and young adulthood.

Adolescence into young adulthood is a vulnerable period for brain development, characterized by profound and 
dynamic changes in cortical areas, the limbic system, and white matter tracts associated with higher order cognitive and affective processes (Bava \& Tapert, 2010). Gray matter volume peaks around the ages of 12 and 14 years and then declines throughout adolescence, first in primary sensorimotor cortices and lastly in the dorsolateral prefrontal cortex (Giedd et al., 1999; Gogtay et al., 2004; Gogtay \& Thompson, 2010; Sowell, Thompson, Holmes, Jernigan, \& Toga, 1999). During adolescence, increased myelination and synaptic pruning occurs (Sowell, Thompson, Tessner, \& Toga, 2001; Huttenlocher \& Dabholkar, 1997), with white matter volume peaking in mid-life (Bartzokis et al., 2010). The endocannabinoid system also changes during this period with significant pruning of receptor density in subcortical and frontal regions (Heng, Beverley, Steiner, \& Tseng, 2011). Brain regions centrally involved in higher-order cognitive functions and with a high expression of cannabinoid receptors (CB1) may thus be particularly susceptible to insults from exogenous cannabinoids (Herkenham et al., 1990; Mackie, 2005; Van Laere et al., 2008). There is evidence that sex moderates the impact of adolescent cannabis exposure on brain structure, with female users aged 16 to 19 years reported to show larger amygdala volume compared with male and female non-users (Mcqueeny et al., 2011). In female users aged 16 to 18 years, larger volume of the prefrontal cortex (PFC) has been reported to have an association with poorer executive functioning specifically (Medina et al., 2009).

Previous research has focused on the effects of heavy cannabis use on neuropsychological functioning in adolescents and young adults, with studies showing small overall effect sizes (Scott et al., 2018). Nonetheless, cognitive difficulties have been shown to improve with abstinence (Schuster et al., 2018; Wallace, Wade, \& Lisdahl, 2020), supporting their association with continued use. In young adults, sex has been reported to moderate the association between cognitive dysfunction and the frequency (Lisdahl \& Price, 2012) and amount (Crane, Schuster, \& Gonzalez, 2013) of cannabis use, as well as the age of initiated use (Crane, Schuster, Mermelstein, \& Gonzalez, 2015). Several studies have supported associations between earlier age of cannabis use onset and worse neuropsychological outcomes (e.g. Battisti et al., 2010; Ehrenreich et al., 1999; Fontes et al., 2011; Gruber, Sagar, Dahlgren, Racine, \& Lukas, 2012; Schuster, Hoeppner, Evins, \& Gilman, 2016). Sex may also moderate the severity of withdrawal from cannabis. For example, females report greater incidence and severity of withdrawal symptoms and experience more difficulty achieving abstinence than males (Cooper \& Craft, 2018; Herrmann, Weerts, \& Vandrey, 2015). However, the impact of sex on the relationship between withdrawal severity and neuropsychological functioning remains to be clarified.

We investigated the relationship between sex, cannabis exposure levels, and cognitive performance using a standardized comprehensive test battery, controlling for key demographic and drug-related variables (i.e. age, IQ, amount of alcohol and nicotine use in the past 90 days, mood and anxiety symptoms, emotional stability, and impulsive behavior) in a community sample of high school and college students aged 13 to 25 years. Based on previous evidence showing sex-specific disruption of CB1 receptor signaling by repeated cannabis exposure (Burston, Wiley, Craig, Selley, \& SimSelley, 2010), prefrontal structural differences between sexes (Medina et al., 2009), and sex differences in neuropsychological functioning among adult cannabis users (e.g. Lisdahl \& Price, 2012; King et al., 2011; Pope et al., 1997), we hypothesized that female adolescents who used cannabis would demonstrate worse cognitive performance than males, particularly for functions subserved by frontallimbic circuitry. In addition, we tested whether sex moderated the relationship between neuropsychological functioning and age of initiation of regular cannabis use, frequency of cannabis use in the past 90 days, amount of cannabis use in the past 90 days, and on cannabis withdrawal symptoms in this sample.

\section{METHOD}

\section{Participants}

This study evaluated the baseline wave of data collection from two longitudinal trials of cannabis use and cognition, one involving participants aged 18-25 years (Cohort $1 n$ $=88 ; 50$ male, 38 female) and one involving those aged 13-19 years (Cohort $2 n=83 ; 42$ male, 41 female), both of whom reported using cannabis at least weekly (i.e. at minimum once per week, most weeks, and at least once within 7 days of baseline). The primary aim of the parent studies is to evaluate the impact of cannabis abstinence on neuropsychological functioning in a community sample of adolescents. Participants were recruited from community advertisements, peer referrals, and school surveys administered in the local Boston community, and screened for eligibility by telephone. Inclusion criteria included English language fluency and at least weekly cannabis use. Participants were excluded for severe developmental delays likely to impact neuropsychological functioning, including but not limited to autism spectrum disorder, intellectual disability, and Down's syndrome. All participants were asked to refrain from all substance use on the day of their study visit, with the exception of nicotine, caffeine, and prescription drug use. Participants over the age of 18 provided written informed consent. For participants under the age of 18, written informed assent was obtained as was written informed consent from a parent or legal guardian. Protocols were approved by the Partners Human Research Committee Institutional Review Board.

\section{MEASURES}

\section{Substance Use}

Frequency of cannabis and alcohol use in the past 90 days (cumulative number of days used), amount of cannabis, alcohol and nicotine use in the past 90 days (total number of 
grams of cannabis, ${ }^{1}$ number of alcoholic drinks and number of cigarettes), and age of initiation were assessed using a modified Timeline Follow-Back method (Robinson et al., 2014). Intensity and negative impact of cannabis withdrawal were assessed with the Cannabis Withdrawal Scale (CWS; Allsop, Norberg, Copeland, Fu, \& Budney 2011; Cronbach's $\alpha=0.91$ ). Cannabis and alcohol dependence were assessed using the Cannabis Use Disorder Identification Test-Revised (CUDIT-R; Adamson \& Sellman, 2003; Cronbach's $\alpha=0.91$ ) and Alcohol Use Disorders Identification Test (AUDIT; Saunders et al., 1993; Cronbach's $\alpha=0.81$ ). Participants provided a urine sample on the day of their study visit to quantitatively ascertain levels of creatinine-adjusted 11-nor-9-carboxy$\Delta$ 9-tetrahydrocannabinol metabolite levels via liquid chromatography-tandem mass spectrometry (LC/MS/MS) and were qualitatively screened with an immunoassay rapid dip drug test (RDDT; Medimpex United Inc.) for the presence of cannabis, cocaine, opiates, amphetamine, and methamphetamine.

\section{Psychopathology}

Current Axis 1 psychiatric disorders (including substance use disorders) were assessed via semi-structured interview (Cohort 1: Structured Clinical Interview for DSM-5, SCID-5; First et al., 2015; and Kiddie Schedule for Affective Disorders and Schizophrenia, K-SADS; Endicott \& Spitzer, 1978; Cohort 2: Mini-International Neuropsychiatric Inventory for Children and Adolescents, MINI Kid; Sheehan et al., 1998). Current and childhood symptoms of attention deficit/hyperactivity disorder (ADHD) were assessed with a DSM-5 symptom checklist (American Psychiatric Association, 2013). Past week symptoms of anxiety and depression were assessed with the Mood and Anxiety Symptom Questionnaire (MASQ; Watson et al., 1995; Cronbach's $\alpha=0.85-0.93$ ).

\section{Trait Measures}

Personality was assessed with the 10-Item Personality Measure (TIPI; Gosling, Rentfrow, \& Swann, 2003; Cronbach's $\alpha=0.40-0.73$ ) and impulsive behavior was assessed with the Urgency, Premeditation (lack of), Perseverance (lack of), Sensation Seeking, Positive Urgency, Impulsive Behavior Scale (UPPS-P; Cyders \& Smith, 2007; Cronbach's $\alpha=0.80-0.94$ ).

\section{Neuropsychological Functioning}

Full-scale IQ was estimated using the Wechsler Test of Adult Reading (WTAR; Wechsler, 1999) in Cohort 1 and

\footnotetext{
${ }^{1}$ Similar to objective methods employed by other research teams (e.g. Hindocha, Freeman, \& Curran, 2017), participants were asked to estimate the typical amount of cannabis used per use occasion. A cannabis placebo sample (i.e. oregano) was weighed to the nearest hundredth gram with a Pocket-Size scale. This estimate was then multiplied by the number of use occasions to estimate the amount of cannabis exposure over the past 90 days.
}

the two-subset Wechsler Abbreviated Scale of Intelligence (WASI; Wechsler, 2001) in Cohort 2. Neuropsychological functioning was assessed using the CANTAB (www. cambridgecognition.com), a reliable and well-validated battery that has been previously used in populations with drug use, including cannabis (e.g. Meier et al., 2012; Grant, Chamberlain, Schreiber, \& Odlaug, 2012). CANTAB Research Suite (Windows-based) was administered in Cohort 1 and CANTAB Connect (iPad-based) was administered in Cohort 2; both platforms are touchscreen, with tasks showing good test-retest reliability (Pearson correlation coefficients $\geq 0.72$ ) (Cambridge Cognition, internal data).

\section{Memory}

The Delayed Matching to Sample (DMS) task assesses visual matching and short-term visual recognition memory (Robbins et al., 1997) (Cohort 1 only). Participants are shown an abstract, non-verbal pattern (the sample) followed by four similar patterns simultaneously and after 4000 and $12000 \mathrm{~ms}$ delays. Participants are then asked to select the pattern that matches the sample. Outcome measures include the percentage of correct responses and mean response latency (ms) at all three delay intervals.

The Paired Associates Learning (PAL) task assesses episodic memory and new learning (Sahakian et al., 1988) (Cohort 2 only). Boxes are displayed on the screen and opened in a randomised order, some of which contain a pattern. The patterns are then displayed in the middle of the screen, one at a time, and the participant must touch the box where they think the pattern was originally located. If a participant makes an error, the patterns are presented again. Outcome measures include the total number of errors made (a measure of new learning) and first trial memory score (i.e. the number of patterns correctly located after the first trial summed across the number of stages completed).

The Verbal Recognition Memory (VRM) task assesses ability to encode and retrieve verbal information (Fletcher $\&$ Henson, 2001) (Cohort 2 only). Eighteen words are presented, and participants are subsequently asked to identify them among distractor words immediately and after a 20 min delay. Outcome measures include the number of correct responses at immediate and delayed recall.

\section{Attention and Executive Functions}

The Rapid Visual Information Processing (RVP) task assesses sustained visual attention (Pironti et al., 2014) (Cohorts 1 and 2). Single digits appear in a white box in the center of the screen at a rate of 100 digits per minute. Participants must detect a series of three target sequences (e.g. $3,5,7$ ) by pressing a button as quickly as possible. Outcome measures include mean response latency (ms) on correctly identified targets and A' (sensitivity to detecting the target sequence regardless of response tendency). 
The Multitasking Test (MTT) assesses ability to manage conflicting information. Arrows appear on either side of the screen (left/right) and point in either direction (to the left or right) (Cohorts 1 and 2). A cue at the top of the screen indicates whether the participant should select the right or left button according to either the side on which the arrow appeared or the direction in which the arrow was pointing. In some sections of the task the same rule is applied consistently (either side or direction, single task), whereas in others the rules are presented in a randomized order (multitasking). Outcome measures include switching cost (the difference between response latencies when the rule was switching vs. when the rule remained constant) and congruency cost (the difference between response latency of congruent $v s$. incongruent trials) for correct responses only.

The One Touch Stockings of Cambridge (OTS) task assesses spatial planning and working memory (Owen, Downes, Sahakian, Polkey, \& Robbins, 1990) (Cohorts 1 and 2). Participants are shown two displays containing three colored balls. Participants must work out the minimum number of moves required to copy the upper display (by moving the balls in the lower display to a new position) and select their response from a row of numbered boxes along the bottom of the screen. Outcome measures include mean choices to correct and the number of problems solved on the first choice.

The Spatial Working Memory (SWM) assesses ability to retain spatial information and manipulate remembered items in working memory (Owen et al., 1990) (Cohort 2). Colored boxes first appear on the screen, and participants are instructed to find a yellow token in each box using a process of elimination. The position of the boxes changes and the number of boxes increases for each trial. Outcome measures include total errors (selecting boxes that have already been found to be empty and/or revisiting boxes which have already been found to contain a token) and strategy (a predetermined sequence by beginning with a specific box and then, once a token has been found, returning to that box to start a new search sequence).

\section{Statistical Analyses}

Analyses were conducted using SPSS 25.0. Demographic, mental health, substance use, and personality trait measures were analyzed by sex using independent samples $t$-tests or chi-square tests as appropriate. Frequency and amount of cannabis and alcohol use were analysed by sex using MannWhitney $U$ tests given their highly skewed distributions. Males and females were compared on CANTAB outcome measures using analysis of covariance for each task separately, controlling for age, estimate of intelligence (IQ), amount of alcohol (number of drinks) and nicotine (number of cigarettes) use in the past 90 days, mood and anxiety symptoms (MASQ subscales), emotional stability (TIPI subscale), and impulsive behavior (UPPS-P perseverance and sensation seeking subscales). The Benjamini-Hochberg procedure was applied at $q<0.10$ to control for false discovery; all significant $p$-values remained (two-sided).

We then explored potential interactions (separately) with age of initiated use, frequency of cannabis use in the past 90 days (number of days), amount of cannabis use in the past 90 days (grams consumed), and withdrawal symptoms to test if similar patterns of cognitive performance were also related to measures of use severity. As in previous studies (Crane, Schuster, Fusar-Poli, et al., 2013; Crane, et al., 2015), we conducted a moderated hierarchical multiple regression analysis with neuropsychological performance as the dependent variable and age of cannabis initiation, frequency of cannabis use, amount of cannabis use, and cannabis withdrawal subscales (intensity, negative impact of withdrawal) as predictors (separately) in Block (model) 1; vectors for sex (male, female) in Block 2; and their interaction in Block 3. We additionally controlled for the same covariates delineated above (entered in the first block) to better isolate any observed effects to the influence of sex. Interdependencies between covariates were examined using multicollinearity diagnostics. Variance inflation factors ranged between 1.1 and 5.4 for each variable and were thus regarded as acceptable.

\section{RESULTS}

\section{Demographic, Mental Health, Trait, and Substance Use Measures}

The groups did not differ significantly in age, IQ, or scholastic achievement. Females reported more than twice the rate of anxiety disorders and significantly greater symptoms on MASQ subscales than males. Males reported greater emotional stability, more perseverance, and higher sensationseeking than females (Table 1).

On substance use measures, males had significantly higher AUDIT scores and a trend for higher CUDIT scores, and reported higher amount of use for alcohol, cannabis and nicotine though not significantly more days using either substance (alcohol or cannabis) or days since last cannabis or nicotine use. Females reported more negative impact of cannabis withdrawal and a trend for greater intensity of withdrawal (Table 2).

\section{Neuropsychological Performance}

Univariate analysis of covariance revealed domain-specific patterns of cognitive performance by sex. Males had slower response latencies (DMS) than females after the 4000 and 12000 delay intervals (i.e. poorer visual recognition memory). Females showed greater switching cost (MTT), poorer target sensitivity (RVP A') (i.e. worse sustained visual attention), and had a less efficient search strategy (SWM) than males (Table 3 ). 
Table 1. Participant characteristics, mental health, substance use and trait measures by sex (means and standard deviations)

\begin{tabular}{|c|c|c|c|}
\hline & $\begin{array}{l}\text { Males } \\
n=92\end{array}$ & $\begin{array}{c}\text { Females } \\
n=79\end{array}$ & Statistic, $p$-value, Effect Size \\
\hline \multicolumn{4}{|l|}{ Demographics/Academic Achievement } \\
\hline Age (Years) & $19.64(2.29)$ & $19.83(2.53)$ & $t(169)=-0.51, p=.61, d=0.08$ \\
\hline Body Mass Index (BMI) & $24.18(3.92)$ & $24.39(5.28)$ & $t(169)=-0.30, p=.77, d=0.05$ \\
\hline Verbal IQ (WTAR/WASI $\left.{ }^{\mathrm{a}}\right)$ & $107.93(9.75)$ & $107.46(12.98)$ & $t(169)=0.28, p=.78, d=0.04$ \\
\hline Handedness (Right/ Left/ Ambidextrous) & $71 / 11 / 4$ & $68 / 6 / 1$ & $x^{2}=2.60, p=.27, V=0.13$ \\
\hline GPA (on a 4.00 scale) & $3.06(0.49)$ & $3.19(0.62)$ & $t(146)=-1.38, p=.17, d=0.23$ \\
\hline \multicolumn{4}{|l|}{ Psychiatric Diagnoses ${ }^{\mathrm{b}}$ (Current) } \\
\hline \% Major Depressive Disorder & $5.4 \%$ & $11.4 \%$ & $x^{2}=2.01, p=.16, V=0.11$ \\
\hline$\%$ Mania & $2.2 \%$ & $2.6 \%$ & $x^{2}=0.02, p=.88, V=0.01$ \\
\hline$\%$ Anxiety Disorder ${ }^{\mathrm{c}}$ & $13.0 \%$ & $29.1 \%$ & $x^{2}=6.74, p=.009, V=0.20$ \\
\hline$\%$ Obsessive-Compulsive Disorder & $2.2 \%$ & $3.8 \%$ & $x^{2}=0.40, p=.53, V=0.05$ \\
\hline$\%$ Eating Disorder & $0 \%$ & $1.3 \%$ & $x^{2}=1.17, p=.28, V=0.08$ \\
\hline \% Psychosis & $1.1 \%$ & $0 \%$ & $x^{2}=0.86, p=.35, V=0.07$ \\
\hline \multicolumn{4}{|l|}{ Psychiatric Symptoms } \\
\hline \multicolumn{4}{|l|}{ Childhood ADHD Symptoms $\left(\max =9^{\mathrm{d}}\right)$} \\
\hline Inattention & $4.00(3.00)$ & $3.25(2.94)$ & $t(169)=1.64, p=.10, d=0.25$ \\
\hline Hyperactivity & $4.32(2.78)$ & $3.61(2.95)$ & $t(169)=1.61, p=.11, d=0.25$ \\
\hline \multicolumn{4}{|l|}{ Current ADHD Symptoms $\left(\max =9^{\mathrm{d}}\right)$} \\
\hline Inattention & $3.50(3.04)$ & $4.35(3.38)$ & $t(169)=-1.74, p=.08, d=0.26$ \\
\hline Hyperactivity & $3.38(2.83)$ & $3.62(2.78)$ & $t(169)=-0.56, p=.58, d=0.09$ \\
\hline \multicolumn{4}{|l|}{ Mood/Anxiety (MASQ) } \\
\hline General Distress Anxious Symptoms $(\max =75)$ & $17.73(5.88)$ & $20.42(7.22)$ & $t(169)=-2.69, p=.008, d=0.41$ \\
\hline Anxious Arousal $(\max =55)$ & $23.77(6.13)$ & $25.87(7.64)$ & $t(169)=-2.00, p=.05, d=0.30$ \\
\hline General Distress Depressive Symptoms $(\max =85)$ & $20.16(7.86)$ & $24.18(9.57)$ & $t(169)=-3.01, p=.003, d=0.46$ \\
\hline Anhedonic Depression $(\max =110)$ & $55.72(12.07)$ & $59.72(12.83)$ & $t(169)=-2.10, p=.04, d=0.32$ \\
\hline \multicolumn{4}{|l|}{ Personality (TIPI) $(\max =14)$} \\
\hline Extraversion & $9.37(3.22)$ & $9.57(2.89)$ & $t(169)=-0.43, p=.67, d=0.07$ \\
\hline Agreeableness & $9.50(2.30)$ & $9.54(2.42)$ & $t(169)=-0.12, p=.90, d=0.02$ \\
\hline Conscientiousness & $9.96(2.37)$ & $9.39(3.34)$ & $t(137.95)=1.25, p=.21, d=0.20$ \\
\hline Emotional Stability & $10.33(2.49)$ & $7.95(3.00)$ & $t(169)=5.67, p<.001, d=0.86$ \\
\hline Openness to Experience & $11.28(1.94)$ & $11.67(2.08)$ & $t(169)=-1.26, p=.21, d=0.19$ \\
\hline \multicolumn{4}{|l|}{ Impulsive Behavior (UPPS-P) } \\
\hline Urgency $(\max =48)$ & $28.12(6.49)$ & $28.52(8.05)$ & $t(149.42)=-0.35, p=.72, d=0.05$ \\
\hline Premeditation $(\max =56)$ & $22.01(4.59)$ & $23.47(6.00)$ & $t(144.83)=-1.76, p=.08, d=0.31$ \\
\hline Perseverance $(\max =40)$ & $20.32(4.73)$ & $21.94(5.71)$ & $t(169)=-2.03, p=.04, d=0.31$ \\
\hline Sensation Seeking $(\max =48)$ & $38.82(5.38)$ & $34.29(6.74)$ & $t(169)=4.88, p<.001, d=0.74$ \\
\hline Positive Urgency $(\max =56)$ & $27.84(8.92)$ & $25.67(9.14)$ & $t(169)=1.57, p=.12, d=0.24$ \\
\hline
\end{tabular}

Notes. WTAR: Wechsler Test of Adult Reading; WASI: Wechsler Abbreviated Scale of Intelligence; MASQ: Mood and Anxiety Symptoms Questionnaire; TIPI: 10-Item Personality Inventory; UPPS-P: Impulsive Behavior Scale.

${ }^{a}$ WTAR/WASI

b Includes SCID-5: Structured Clinical Interview for DSM-5; K-SADS: Schedule for Affective Disorders and Schizophrenia for School Aged Children; and MINI-Kid: MINI-International Neuropsychiatric Interview.

c Anxiety disorders: Gernalized Anxiety Disorder, Posttraumatic Stress Disorder, Panic Disorder, Agoraphobia, and Specific Phobia.

${ }^{\mathrm{d}}$ A score of greater than or equal to 5 indicates symptoms consistent with ADHD.

\section{Relationship between Sex, Age of Initiated Use, and Neuropsychological Performance}

For switching cost on the multitasking test of attention (MTT), there were main effects of age, $[\beta=0.22, p=.02$, $95 \% \mathrm{CI}(1.54,21.52)]$ and sex, $[\beta=1.43, p=.02,95 \% \mathrm{CI}$ $(61.77,649.39)]$ (see Table 4 for main effects and interactions on all neuropsychological outcomes). The interaction between sex and age of cannabis initiation did not reach significance, $\quad\left[R^{2}=.21, \quad \beta=-1.13, \quad p=.07, \quad 95 \% \quad \mathrm{CI}\right.$ $(-36.60,1.12)]$ (Figure 1$)$. In analyses of simple slopes, earlier age of first cannabis use was associated with greater switching cost for females, $[\beta=-0.30, p=.04,95 \% \mathrm{CI}$ $(-36.53,-1.23)]$, but not for males, $[\beta=0.02, p=.88$, $95 \%$ CI $(-13.41,15.62)]$. There was no significant interaction of age of cannabis initiation with sex for other neuropsychological outcomes ( $p$-values $>.11$ ). 
Table 2. Substance use measures (means and standard deviations) by sex

\begin{tabular}{|c|c|c|c|}
\hline & $\begin{array}{l}\text { Males } \\
n=92\end{array}$ & $\begin{array}{c}\text { Females } \\
n=79\end{array}$ & Statistic, $p$-value, Effect Size \\
\hline \multicolumn{4}{|l|}{ Alcohol Use } \\
\hline Age of Initiation (Years) & $14.90(1.89)$ & $15.35(1.85)$ & $t(166)=-1.54, p=.13, d=0.24$ \\
\hline Dependence Symptoms (AUDIT) & $7.82(6.08)$ & $6.04(4.55)$ & $t(166.04)=2.18, p=.03, d=0.33$ \\
\hline Alcohol Dependence (\% Current) & $27.2 \%$ & $19.0 \%$ & $x^{2}=1.59, p=.21, V=0.10$ \\
\hline Days Alcohol Consumed (past 90 days) & $16.65(15.06)$ & $14.23(13.87)$ & $U=3322.50, p=.34, d=0.17$ \\
\hline Drinks Consumed (past 90 days) & $95.15(112.78)$ & $54.27(66.05)$ & $U=2832.50, p=.01, d=0.44$ \\
\hline \multicolumn{4}{|l|}{ Cannabis Use } \\
\hline Age of Initiation (Years) & $15.13(1.97)$ & $15.50(2.03)$ & $t(169)=-1.21, p=.23, d=0.18$ \\
\hline Age of Regular Use (Years) & $16.63(2.04)$ & $17.13(2.14)$ & $t(155)=-1.41, p=.16, d=0.24$ \\
\hline Dependence Symptoms (CUDIT-R) & $14.51(5.27)$ & $13.11(5.30)$ & $t(169)=1.72, p=.09, d=0.27$ \\
\hline Cannabis Dependence (\% Current) & $46.7 \%$ & $45.6 \%$ & $x^{2}=0.02, p=0.88, V=0.01$ \\
\hline $\mathrm{CN}-\mathrm{THCCOOH}{ }^{\mathrm{a}}$ & $230.11(340.65)$ & $215.38(457.46)$ & $U=2480.50, p=.07, d=0.04$ \\
\hline Days Cannabis Consumed (past 90 days) & $59.37(26.21)$ & $47.90(25.91)$ & $U=3084.50, p=.18, d=0.44$ \\
\hline Grams Consumed (past 90 days) & $80.38(103.99)$ & $73.73(135.52)$ & $U=2796.00, p=.009, d=0.06$ \\
\hline Days Since Last Use & $1.54(1.65)$ & $1.96(2.66)$ & $t(169)=-1.26, p=.21, d=0.19$ \\
\hline \multicolumn{4}{|l|}{ Lifetime (Cumulative) Cannabis Use } \\
\hline$<10$ times & $0.02 \%$ & $0 \%$ & \\
\hline 10-50 times & $0.05 \%$ & $17.7 \%$ & \\
\hline 51-100 times & $40.2 \%$ & $43.0 \%$ & $x^{2}=9.73, p=.05, V=0.05$ \\
\hline $101-500$ times & $27.1 \%$ & $24.1 \%$ & \\
\hline 501-100 times & $25.0 \%$ & $15.2 \%$ & \\
\hline \multicolumn{4}{|l|}{ Withdrawal Symptoms } \\
\hline Intensity (CWS) & $29.71(24.04)$ & $36.24(25.40)$ & $t(168)=-1.72, p=.09, d=0.26$ \\
\hline Negative Impact (CWS) & $26.89(19.33)$ & $34.28(24.59)$ & $t(145.10)=-2.15, p=.03, d=0.33$ \\
\hline \multicolumn{4}{|l|}{ Nicotine Use } \\
\hline Cigarettes Consumed (past 90 days) & $69.00(186.09)$ & $6.92(32.06)$ & $U=2412.00, p<.001, d=0.46$ \\
\hline Days Since Last Use & $18.05(51.25)$ & $24.81(52.40)$ & $t(129)=-0.73, p=.46, d=0.19$ \\
\hline
\end{tabular}

Notes. AUDIT: Alcohol Use Disorders Identification Test; CUDIT-R: Cannabis Use Disorder Identification Test Revised; CWS: Cannabis Withdrawal Scale. ${ }^{\mathrm{a}}$ Creatinine-adjusted 11-nor-9-carboxy- $\Delta$-tetrahydrocannabinol in $\mathrm{ng} / \mathrm{ml}$.

\section{Relationship between Sex, Frequency and Amount of Cannabis Use, and Neuropsychological Performance}

There were no significant interactions of frequency of cannabis use in the past 90 days with sex for neuropsychological outcomes ( $p$-values $>.18$ ). For mean response latency on the task of sustained visual attention (RVP), there was a main effect of age, $[\beta=-0.27, p<.001,95 \%$ CI (597.06, 1143.49)], and the interaction between sex and amount of cannabis use in the past 90 days was significant, $\left[R^{2}=.24\right.$, $\beta=0.28, p=.02,95 \% \mathrm{CI}(0.05,0.55)]$ (Figure 2). In analyses of simple slopes, more cannabis use was associated with slower response latency for females, $[\beta=0.35, p=.004$, $95 \% \mathrm{CI}(0.92,0.45)]$, but not for males, $[\beta=0.04, p=.72$, 95\% CI $(-0.19,0.27)]$.

For the PAL first trial memory score, there were main effects of sex, $[\beta=-0.48, p=.002,95 \%$ CI $(-4.84$, $-1.10)]$ and amount of use, $[\beta=-0.64, p=.001,95 \% \mathrm{CI}$ $(-0.02,-0.01)]$, and their interaction was significant, $\left[R^{2}=.29, \quad \beta=0.68, \quad p=.001, \quad 95 \% \quad\right.$ CI $\left.(0.01,0.03)\right]$ (Figure 3A). Similarly for PAL total errors made, there were main effects of sex, $[\beta=0.46, p=.002,95 \% \mathrm{CI}(2.18,8.84)]$ and amount of use, $[\beta=0.88, p<.001,95 \% \mathrm{CI}(0.02,0.05)]$, and their interaction was also significant, $\left[R^{2}=.40\right.$, $\beta=-0.78, p<.001,95 \%$ CI $(-0.05,-0.02)]$ (Figure 3B). Analyses of the simple slopes showed that more cannabis use was more strongly associated with worse episodic memory in males [memory score: $\beta=-0.61, p<.001$, $95 \%$ CI $(-0.02,-0.01)$ ] than in females [memory score: $\beta=0.42, p=.04,95 \%$ CI $(0.00,0.01)]$. More cannabis use was associated with poorer learning (total errors) in males only [males: $\beta=0.72, p<.001,95 \%$ CI $(0.03,0.05)$ ]; [females: $\beta=-0.24, p=.22,95 \%$ CI $(-0.02,0.004)$ ].

\section{Relationship between Sex, Withdrawal, and Neuropsychological Performance}

For OTS mean choices to correct, there were main effects of IQ, $[\beta=-0.33, p<.001,95 \%$ CI $(-0.01,-0.01)]$ and anxious arousal, $[\beta=0.32, p=.01,95 \% \mathrm{CI}(0.004,0.02)]$, and the interaction between sex and negative impact of cannabis withdrawal was significant. $\left[R^{2}=.24, \beta=0.40, p=.02,95 \%\right.$ CI $(0.001,0.009)]$. For OTS number of problems solved, there were main effects of IQ, $[\beta=0.19, p=.03,95 \% \mathrm{CI}$ $(0.004,0.07)]$, cigarette use in the past 90 days, $[\beta=-0.20, p=.01,95 \%$ CI $(-0.01,-0.001)]$, anxious 
Table 3. Neuropsychological outcome measures (means and standard deviations) by sex

\begin{tabular}{|c|c|c|c|}
\hline & $\begin{array}{l}\text { Males } \\
n=92\end{array}$ & $\begin{array}{c}\text { Females } \\
n=79\end{array}$ & Statistic, $p$-value, Effect Size \\
\hline \multicolumn{4}{|l|}{ Memory } \\
\hline \multicolumn{4}{|l|}{ Delayed Matching to Sample (DMS) ${ }^{\mathrm{a}}$} \\
\hline$\%$ Correct (Average of Delays) & $70.40(8.80)$ & $66.71(13.37)$ & $F(1,75)=2.70, p=.11, \eta_{p}^{2}=0.04$ \\
\hline Correct Latency (simultaneous) & $2829.92(700.44)$ & $2376.40(627.03)$ & $F(1,75)=3.80, p=.06, \eta_{p}^{2}=0.05$ \\
\hline Correct Latency 4000 (delay) ms & $3624.86(976.37)$ & $2938.86(861.65)$ & $F(1,75)=6.80, p=.01, \eta_{p}{ }^{2}=0.08$ \\
\hline Correct Latency 12000 (delay) ms & $4038.74(1206.03)$ & $3262.20(1012.71)$ & $F(1,75)=8.67, p=.004, \eta_{p}{ }^{2}=0.10$ \\
\hline \multicolumn{4}{|l|}{ Paired Associates Learning (PAL) ${ }^{\mathrm{b}}$} \\
\hline Total Errors (Adjusted) & $5.40(6.90)$ & $6.90(4.71)$ & $F(1,70)=1.21, p=.28, \eta_{p}^{2}=0.02$ \\
\hline First Trial Memory Score & $16.29(3.21)$ & $15.02(2.91)$ & $F(1,70)=2.33, p=.13, \eta_{p}^{2}=0.03$ \\
\hline \multicolumn{4}{|l|}{ Verbal Recognition Memory $(\mathrm{VRM})^{\mathrm{b}}$} \\
\hline$\%$ Total Correct (Immediate) & $32.69(3.16)$ & $33.17(3.15)$ & $F(1,70)=0.14, p=.71, \eta_{p}^{2}=0.02$ \\
\hline$\%$ Total Correct (Delayed) & $32.26(2.80)$ & $33.22(2.06)$ & $F(1,70)=1.43, p=.24, \eta_{p}^{2}=0.02$ \\
\hline \multicolumn{4}{|l|}{ Attention/Executive Functions } \\
\hline \multicolumn{4}{|l|}{ Multitasking Test $(\mathrm{MTT})^{\mathrm{c}}$} \\
\hline Switching Cost ms & $160.12(113.11)$ & $228.14(127.50)$ & $F(1,147)=12.23, p=.001, \eta_{p}^{2}=0.08$ \\
\hline Congruency Cost ms & $43.22(44.14)$ & $41.70(39.23)$ & $F(1,147)=3.44, p=.07, \eta_{p}^{2}=0.02$ \\
\hline \multicolumn{4}{|c|}{ Rapid Visual Information Processing $(\mathrm{RVP})^{\mathrm{c}}$} \\
\hline $\mathrm{A}^{\prime}$ & $0.93(0.05)$ & $0.91(0.05)$ & $F(1,157)=6.52, p=.01, \eta_{p}^{2}=0.04$ \\
\hline Latency ms & $443.75(106.64)$ & $474.79(109.04)$ & $F(1,157)=2.41, p=.12, \eta_{p}^{2}=0.02$ \\
\hline \multicolumn{4}{|c|}{ One Touch Stockings of Cambridge $(\mathrm{OTS})^{\mathrm{c}}$} \\
\hline Choices to Correct & $1.35(0.26)$ & $1.44(0.33)$ & $F(1,158)=3.43, p=.07, \eta_{p}{ }^{2}=0.02$ \\
\hline Problems Solved & $11.50(2.01)$ & $11.01(2.18)$ & $F(1,158)=3.11, p=.08, \eta_{\mathrm{p}}{ }^{2}=0.02$ \\
\hline \multicolumn{4}{|l|}{ Spatial Working Memory $(\mathrm{SWM})^{\mathrm{b}}$} \\
\hline Total Errors & $4.69(6.77)$ & $7.14(7.87)$ & $F(1,70)=3.71, p=.06, \eta_{p}^{2}=0.05$ \\
\hline Strategy & $5.29(2.63)$ & $6.88(2.45)$ & $F(1,70)=7.67, p=.01, \eta_{p}^{2}=0.10$ \\
\hline
\end{tabular}

Notes. Analyses are controlling for (as covariates): age, estimate of intelligence (IQ), amount (quantity) of alcohol and nicotine use in the past 90 days, mood and anxiety symptoms (MASQ subscales), emotional stability (TIPI subscale), and impulsive behavior (UPPS-P perseverance and sensation seeking subscales). ${ }^{\text {a }}$ Cohort $1, n=88$ (50 male, 38 female).

b Cohort 2, $n=83$ (42 male, 41 female).

${ }^{\mathrm{c}}$ Cohorts 1 and 2, $n=171$ (79 male, 92 female).

arousal, $[\beta=-0.32, p=.01,95 \%$ CI $(-0.17,-0.02)]$, and perseverance, $[\beta=0.22, p=.02,95 \% \mathrm{CI}(0.02,0.16)]$, and the interaction between sex and negative impact of cannabis withdrawal was significant, $\left[R^{2}=.20, \beta=-0.40, p=.02\right.$, $95 \%$ CI $(-0.06,-0.01)]$. However, analyses of the simple slopes did not support sex as a notable factor ( $p$-values $>$ .34). There were no significant sex by withdrawal intensity interactions for neuropsychological outcomes $(p$-values $>.11)$.

\section{DISCUSSION}

In a community sample of adolescent and young adult regular cannabis users, we found domain-specific patterns of cognitive performance by sex, such that males showed poorer visual recognition memory and females showed worse performance on measures of attention (sustained visual, multitasking) and executive functions (i.e. spatial planning/ working memory subdomains). Exploratory analyses further suggested that earlier age of initiated use was associated with poorer ability to multitask for females, but not for males, and that more cannabis use was associated with slower response latency for females, but not for males. Exploratory analyses also showed that sex interacted with cannabis use in the past 90 days to moderate memory performance. Specifically, more cannabis use was more strongly associated with worse episodic memory (i.e. the total number of patterns correctly located after the first trial) in males than in females. More use was associated with worse learning (i.e. the total number of errors made across all trials attempted) in males, but not females.

\section{Domain-Specific Sex Differences in Neuropsychological Performance}

Between-group comparisons in our sample suggested that sex differences in neuropsychological functioning are domain specific. Nonetheless, we can only draw conclusions about our cannabis-using sample by sex and cannot compare performance relative to non-users. Previous studies examining sex differences in neuropsychological functioning have found that in general, healthy, non-cannabis using males show better decision-making, spatial processing, and sensorimotor/ motor speed (e.g. Reavis \& Overman, 2001; Gur et al., 
Table 4. Main effects and interactions of age of cannabis initiation, amount of use, frequency of use, and withdrawal symptoms on neuropsychological outcome measures $(\beta, p$-values and $95 \%$ confidence intervals)

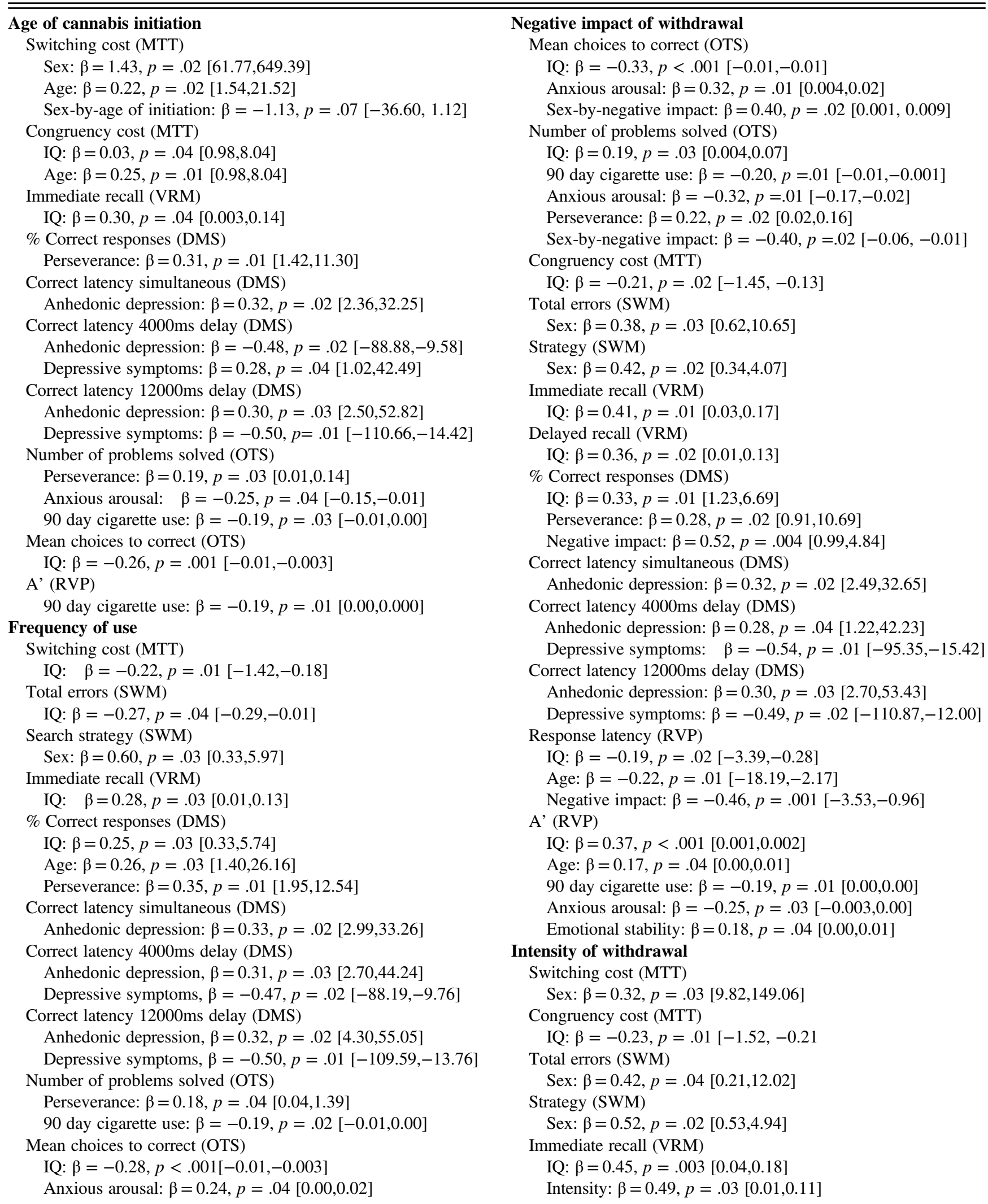


Table 4. (Continued)

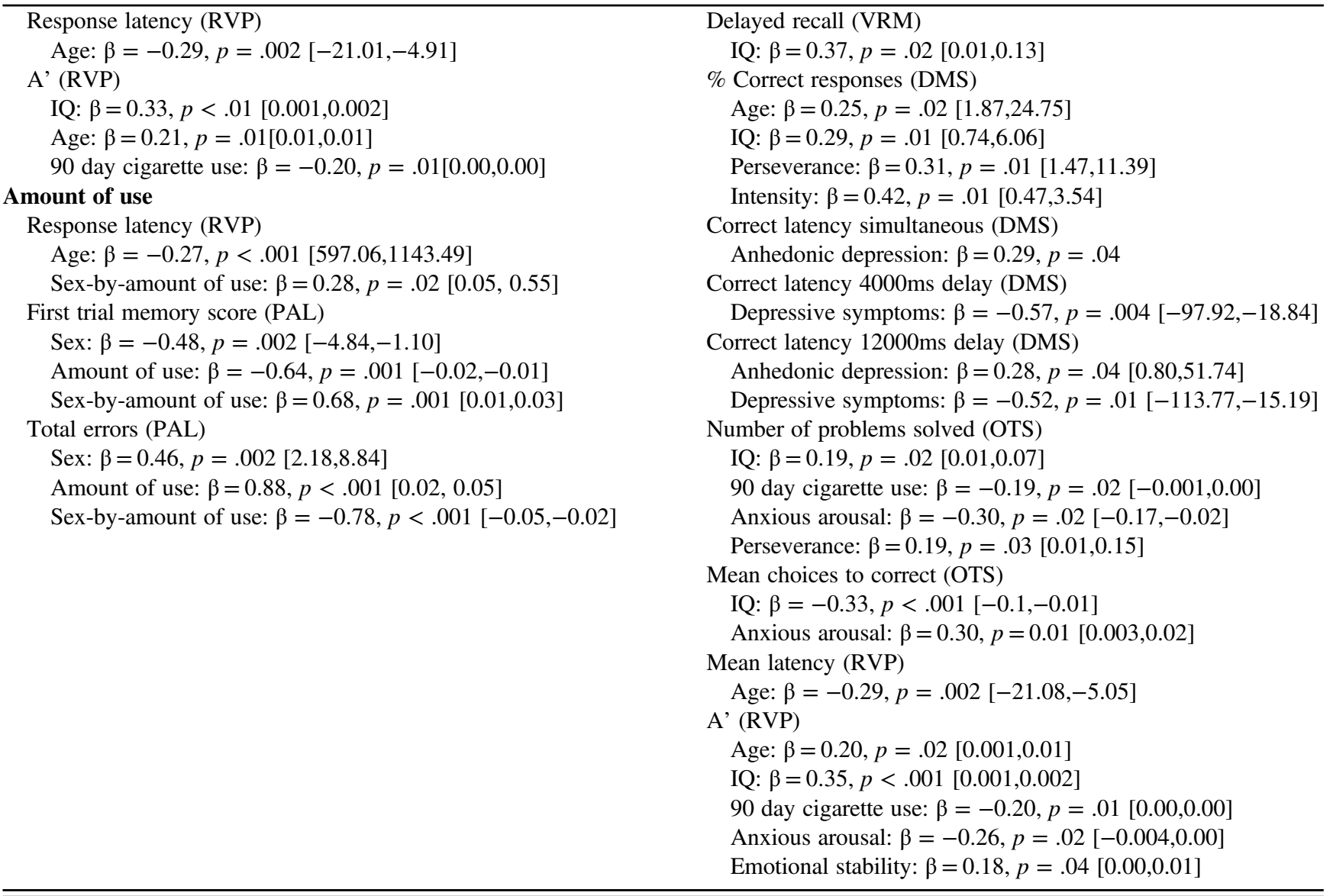

Notes: DMS: Delayed Matching to Sample; PAL: Paired Associates Learning; VRM: Verbal Recognition Memory; MTT: Multitasking Test; RVP: Rapid Visual Information Processing; OTS: One Touch Stockings of Cambridge; SWM: Spatial Working Memory

2012), and healthy, non-cannabis using females show better attention, reasoning, verbal and episodic memory (Gur et al., 2010; Kramer, Delis, \& Daniel, 1988; Asperholm, Högman, Rafi, \& Herlitz, 2019). A reverse pattern of cognitive performance was partly found in our study, such that female cannabis users showed poorer attentional functions. Similar sex-specific patterns of reverse association have been previously reported in non-acute studies, with females showing poorer episodic memory and males showing poorer decision-making, suggesting that cannabis use may blunt those domains in which healthy males and females typically perform better (Crane, Schuster, Mermelstein, et al., 2015).

\section{Attentional Dysfunction in Female Cannabis Users}

Our data provide some support of prior studies showing a negative relationship between earlier age of initiated use and poorer neuropsychological functioning, including measures of attention (e.g. Battisti et al., 2010; Ehrenreich et al., 1999; Fontes et al., 2011; Gruber, Sagar, Dahlgren, Racine, \& Lukas, 2012). Others have reported that attentional problems persist only in female users in early adolescence (i.e. $<17$ years) even after controlling for time-varying confounds (Pardini et al., 2015). In our study, switching cost
(MTT) was significantly worse for females than for males, although the interaction between sex and age of initiation did not reach significance. However, more cannabis use was highly associated with slower response latency (RVP) for females only, indicating that greater cannabis exposure specifically interacts with a key indicator of sustained attentional function. Although attentional problems in early development are more characteristic of healthy, non-cannabis using males, we speculate that self-treatment of potentially undiagnosed attentional problems may drive earlier and greater use in cannabis-using females (current inattention trended toward, but did not reach, significance in the current sample).

Our findings suggested sex-specific disruption to frontal-limbic circuitry most affected by cannabis use (Crane, Schuster, \& Gonzalez, 2013; Martin-Santos et al., 2010). Worse attention in females is consistent with both human and animal studies. For example, preclinical evidence has shown that females selectively metabolize THC to its most active compound (Narimatsu, Watanabe, Yamamoto, \& Yoshimura, 1991) and show widespread desensitization of CB1 receptors in key areas including the PFC (Burston et al, 2010). Neuroimaging evidence has shown PFC-related executive dysfunction 


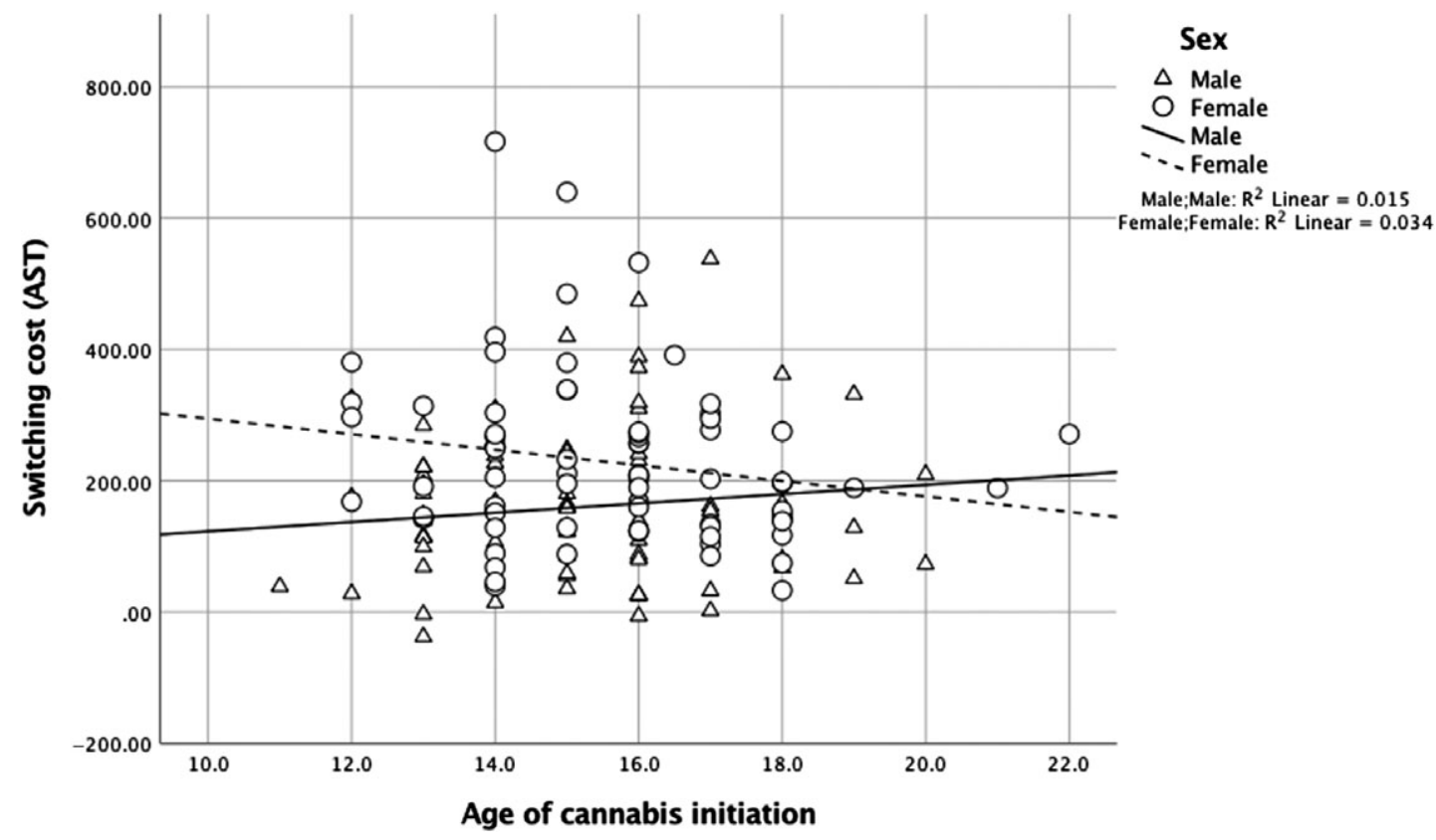

Fig. 1. For switching cost (MTT), the interaction between sex and age of cannabis initiation did not reach significance, $\left[R^{2}=.21, \beta=-1.13\right.$, $p=.07,95 \% \mathrm{CI}(-36.60,1.12)]$. Earlier age of first cannabis use was associated with greater switching cost for females, $[\beta=-0.30, p=.04$, $95 \%$ CI $(-36.53,-1.23)]$, but not for males, $[\beta=0.02, p=.88,95 \%$ CI $(-13.41,15.62)]$.

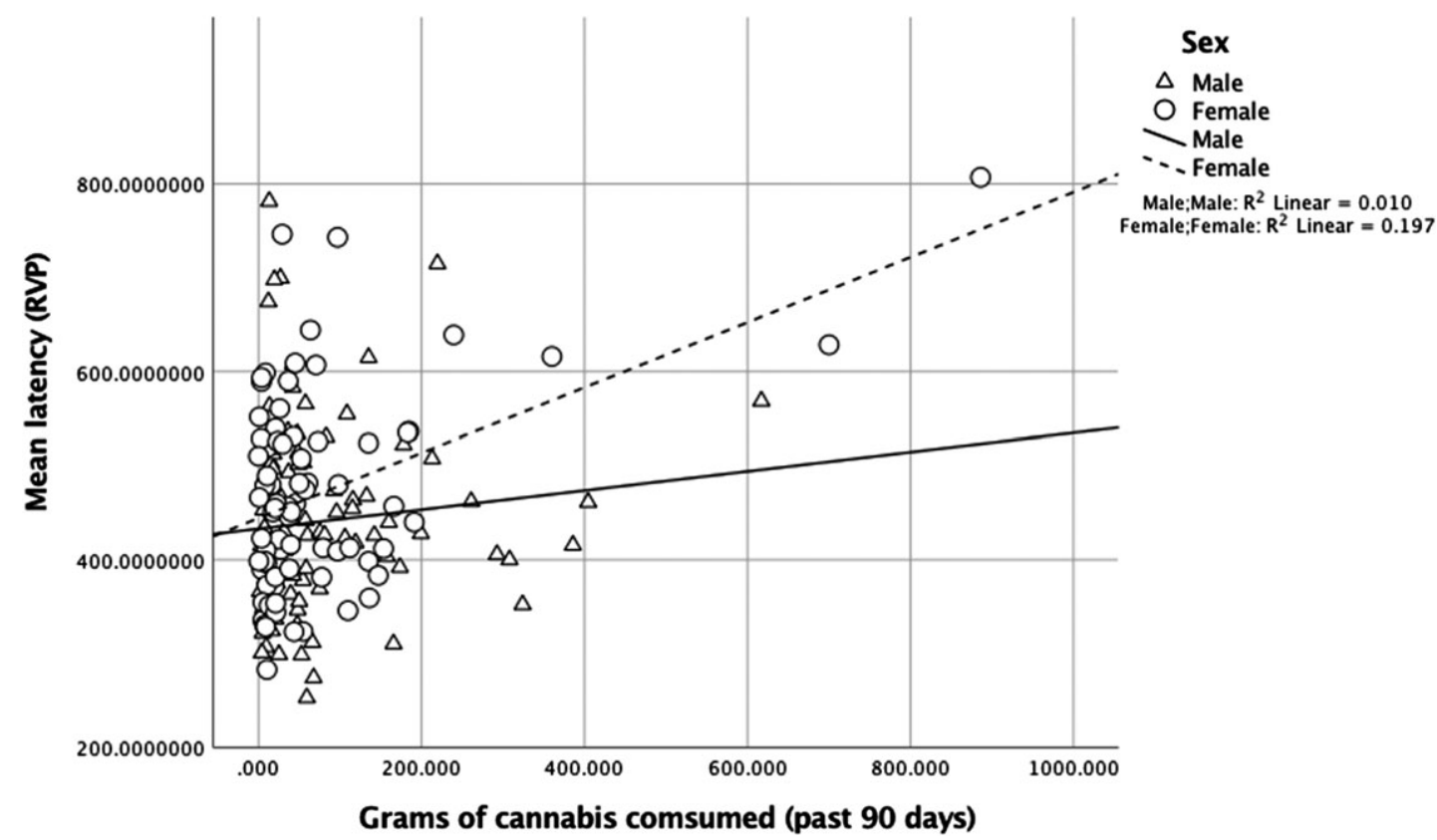

Fig. 2. For mean response latency (RVP), the interaction between sex and amount of cannabis use in the past 90 days was significant, $\left[R^{2}=.24, \beta=0.28, p=.02,95 \% \mathrm{CI}(0.05,0.55)\right]$. More use was associated with slower response latency for females, $[\beta=0.35, p=.004$, $95 \% \mathrm{CI}(0.92,0.45)]$, but not for males, $[\beta=0.04, p=.72,95 \% \mathrm{CI}(-0.19,0.27)]$.

specifically in females (Medina et al., 2009). Neuromaturation occurs in a "back to front" direction, such that the PFC is among the most susceptible regions to repeated exogeneous cannabinoid exposure during development (Gogtay \& Thompson, 2010). As PFC volume peaks 1 to 2 years earlier in females (Giedd et al., 1999), it seems tenable that males might perform cognitively worse than females, given their protracted development of higher-order prefrontal areas. However, others have reported disruption to gray matter pruning in females (Medina et al., 2009). Interruption to pruning at later stages of development, combined with higher availability of $\mathrm{CB} 1$ receptors in similar regions that increase with age (Van Laere et al., 2008), might underlie the sex differences observed here. 

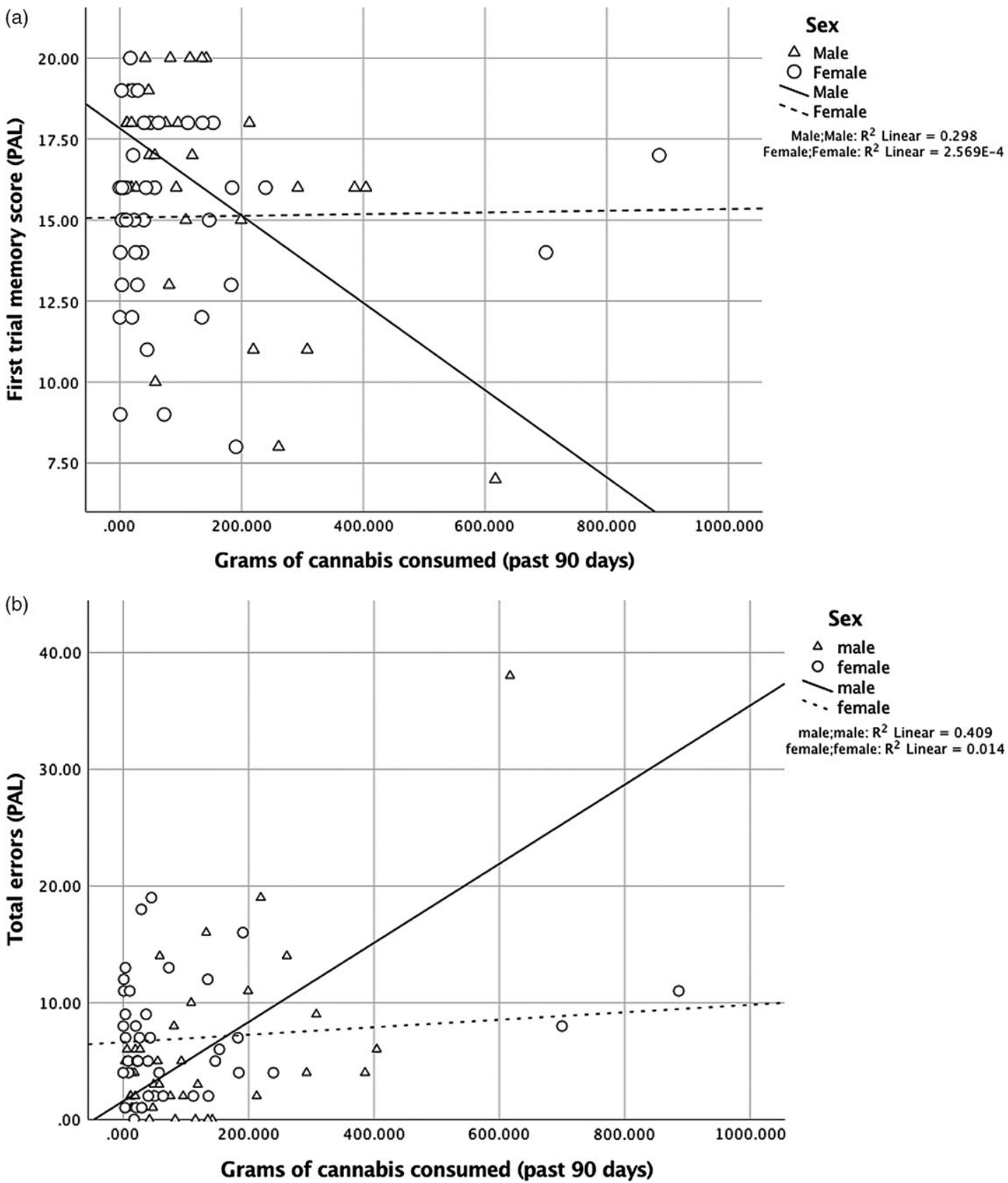

Fig. 3. (A) For first trial memory score (PAL), the interaction between sex and amount of cannabis use in the past 90 days was significant, $\left[R^{2}=.29, \beta=0.68, p=.001,95 \% \mathrm{CI}(0.01,0.03)\right]$. More use was more strongly associated with worse episodic memory in males $[\beta=-0.61$, $p<.001,95 \% \mathrm{CI}(-0.02,-0.01)]$ than in females $[\beta=0.42, p=.04,95 \% \mathrm{CI}(0.00,0.01)]$. (B) For total errors (PAL), the interaction between sex and amount of cannabis use in the past 90 days was significant, $\left[R^{2}=.40, \beta=-0.78, p<.001,95 \%\right.$ CI $\left.(-0.05,-0.02)\right]$. More use was associated with poorer learning (total errors) for males $[\beta=0.72, p<.001,95 \% \mathrm{CI}(0.03,0.05)]$, but not for females $[\beta=-0.24, p=.22,95 \%$ CI $(-0.02,0.004)]$.

The pattern of results was the same when removing cases more than $3 * \mathrm{IQR}$ (interquartile range) (memory score: males: $\beta=0.42, p=.02$, females: $\beta=0.42, p=.04$; errors: males: $\beta=0.39, p=.04$, females: $\beta=-0.24, p=.22$ )

\section{Association between Amount of Cannabis Use and Memory Function}

Sex significantly interacted with amount of cannabis use to moderate memory performance. Specifically, we found that more use was more strongly associated with worse episodic memory in males than in females. More use was also associated with poorer learning in males, but not females. For both PAL measures (memory score, total errors), the interaction between sex and amount of use was highly significant and without influence from confounding variables, indicating sex-specific effects. These findings replicate previous studies reporting poorer memory processes with more use 
(e.g. Bolla, Brown, Eldreth, Tate, \& Cadet, 2002; Solowij et al., 2002). With respect to sex differences, a previous study reported that greater lifetime cannabis use was more strongly associated with poorer episodic memory in females than in males (Crane, Schuster, Fusar-Poli, et al., 2013). Another study reported an association between earlier age of initiated use and poorer episodic memory, particularly immediate recall, for females only (Crane et al., 2015). It is important to note that both of these studies assessed verbal episodic memory (using the Hopkins Verbal Learning Test-Revised; Benedict, Schretlen, Groninger, \& Brandt, 1998) rather than visual. Sex differences in verbal recognition memory (VRM) were not found in our sample. This could be due to differences between the tasks used, such as word lengths, semantic categorization, the number of learning trials, and/ or the method of delivery (i.e. words presented verbally $v s$. read on a computer screen). Nonetheless, our findings suggest that the interaction between cannabis exposure and visual episodic memory differentially affects males and females, thus highlighting the need for sex-specific behavioral interventions, such as those that explicitly target new learning in males.

\section{Strengths, Limitations and Future Directions}

As findings from adult studies are unlikely to generalize to younger populations, our study evaluated sex differences in neuropsychological functioning in a large, generalizable sample of adolescent and young adult regular cannabis users. We also carefully controlled for a wider array of covariates than in prior studies to better isolate the role of sex. Perhaps most importantly, including a similar proportion of male and female participants allowed for critical examination of sex-moderated neuropsychological differences in line with NIDA-supported drug research initiatives (Wetherington, 2007).

The main limitation of our study is the lack of a non-using control group. As such, it is not possible to determine if cognitive performance in our sample was weaker relative to nonusers. It is also not possible from the cross-sectional design to determine whether sex differences in neuropsychological functioning predated or emerged as a consequence of regular cannabis use. Similarly, we cannot draw conclusions about the long-term effects of sex on neuropsychological functioning. We also did not exclude history of neurological illnesses (unless it precluded safe participation, e.g. epilepsy) or traumatic brain injury, which could affect cognitive performance. Finally, we acknowledge that despite controlling for mood and anxiety symptoms, this may not fully account for confounding effects of current anxiety disorders among female users on attentional performance.

Avenues of additional research include defining the hormonal, metabolic, and neuronal mediators of the observed sex differences in neuropsychological functioning. Future research should also explore if sex differences in neuropsychological functioning are more exaggerated in non-acute compared with acute samples, and whether they persist, improve, or reverse with abstinence. Following the long-term implications of these differences is of clinical significance to determine, for example, if they predict cannabis-use trajectories over time, transition to addiction, and/or treatment response. Longitudinal exploration of cognitive functioning and its relationship with age of initiation, severity of use, and withdrawal symptoms would also have strong practical utility.

\section{CONCLUSIONS}

As cannabis legalization becomes more normative, it is important to understand its influence on the developing brain, including potential harmful effects. Our study showed that sex differences in neuropsychological functioning are domain-specific, which may reflect sex-related disruptions in frontal-limbic circuitry that emerge prior to or as a result of regular, early-onset cannabis use. Larger studies including healthy controls are needed to confirm the specificity of these effects to cannabis use, their causality, and critically, whether the neuropsychological outcomes observed in our sample are weaker compared with non-users. Sex-specific behavioral interventions may be beneficial for young cannabis users, including those that take into account dimorphic cognitive outcomes as well as diverse reasons for initiated use. As neuromaturation continues into the third decade of life, longitudinal and multimodal neuroimaging studies investigating the differential impact of cannabis on neuropsychological sequelae from pre-adolescence to young adulthood are needed in males and females separately.

\section{ACKNOWLEDGEMENTS}

Protocols were approved by the Partners Human Research Committee Institutional Review Board.

\section{FUNDING}

This publication was made possible by support from NIHNIDA (1K23DA042946, Schuster; K24 DA030443, Evins), Harvard Medical School (Norman E. Zinberg Fellowship in Addiction Psychiatry and Livingston Fellowship; Schuster), the Massachusetts General Hospital Executive Committee on Research (ECOR; Claflin Distinguished Scholar Award; Schuster), and the Department of Psychiatry at the Massachusetts General Hospital (Louis V. Gerstner III Research Scholar Award; Schuster). George Savulich was funded by Eton College and The Wallitt Foundation, with support from the NIHR Cambridge Biomedical Research Centre (BRC) Mental Health Theme. Barbara J Sahakian's research is conducted within the NIHR MedTech and in vitro diagnostic Co-operative (MIC) and the NIHR Cambridge Biomedical Research Centre (BRC) Mental Health Theme. 


\section{CONFLICTS OF INTEREST}

BJS consults for Cambridge Cognition, Greenfield BioVentures and Cassava Sciences. AEE is a co-founder of Brain Solutions, LLC. AEE has received research grant support to her institution from Charles River Analytics, Pfizer Inc., Forum Pharmaceuticals, and GSK, and honoraria for DSMB or advisory board work from Karuna Pharmaceuticals, Pfizer, and Alkermes in the past 5 years for work unrelated to this project. All other authors have no conflicts of interest.

\section{REFERENCES}

Adamson, S.J. \& Sellman, J.D. (2003). A prototype screening instrument for cannabis use disorder: The Cannabis Use Disorders Identification Test (CUDIT) in an alcohol-dependent clinical sample. Drug and Alcohol Review, 22, 309-315. doi: 10.1080/ 0959523031000154454

Allsop, D.J., Norberg, M.M., Copeland, J., Fu, S., \& Budney, A.J. (2011). The Cannabis Withdrawal Scale development: Patterns and predictors of cannabis withdrawal and distress. Drug and Alcohol Dependence, 119, 123-129. doi: 10.1016/j.drugalcdep. 2011.06.003

American Psychiatric Association. (2013). Diagnostic and Statistical Manual of Mental Disorders (5th ed.). Washington, DC: Publisher.

Asperholm, M., Högman, N., Rafi, J., \& Herlitz, A. (2019). What did you do yesterday? A meta-analysis of sex differences in episodic memory. Psychological Bulletin, 145, 785-821. doi: 10. 1037/bul0000197

Bartzokis, G., Lu, P.H., Tingus, K., Mendez, M.F., Richard, A., Peters, D.G., ... Mintz, J. (2010). Lifespan trajectory of myelin integrity and maximum motor speed. Neurobiology of Aging, 31, 1554-1562. doi: 10.1016/j.neurobiolaging.2008.08.015

Battisti, R.A., Roodenrys, S., Johnstone, S.J., Pesa, N., Hermens, D.F., \& Solowij, N. (2010). Chronic cannabis users show altered neurophysiological functioning on Stroop task conflict resolution. Psychopharmacology, 212, 613-624. doi: 10.1007/s00213-010-1988-3

Bava, S. \& Tapert, S.F. (2010). Adolescent brain development and the risk for alcohol and other drug problems. Neuropsychology Review, 20, 398-413. doi: 10.1007/s11065-010-9146-6

Benedict, R.H.B., Schretlen, D., Groninger, L., \& Brandt, J. (1998). Hopkins verbal learning test revised: Normative data and analysis of inter-form and test-retest reliability. Clinical Neuropsychologist, 12, 43-55.

Bolla, K.I., Brown, K., Eldreth, D., Tate, K., \& Cadet, J.L. (2002) Dose-related neurocognitive effects of marijuana use. Neurology, 59, 1337-1343. doi: 10.1212/01.wnl.0000031422.66442.49

Burston, J.J., Wiley, J.L., Craig, A.A., Selley, D.E., \& Sim-Selley, L.J. (2010). Regional enhancement of cannabinoid $\mathrm{CB}_{1}$ receptor desensitization in female adolescent rats following repeated Delta-tetrahydrocannabinol exposure. British Journal of Pharmacology, 161, 103-112. doi: 10.1111/j.1476-5381.2010. 00870.x

Clark, L., Roiser, J.P., Robbins, T.W., \& Sahakian, B.J. (2009). Disrupted 'reflection' impulsivity in cannabis users but not current or former ecstasy users. Journal of Psychopharmacology, 23, 14-22. doi: 10.1177/0269881108089587
Cohen, P.J (2010). Medical marijuana 2010: It's time to fix the regulatory vacuum. The Journal of Law, Medicine, \& Ethics, 38, 654-666. doi: 10.1111/j.1748-720X.2010.00519.x

Cooper, Z.D., \& Craft, R.M. (2018). Sex-dependent effects of cannabis and cannabinoids: A translational perspective. Neuropsychopharmacology, 43, 34-51. doi: 10.1038/npp.2017.140

Crane, N.A., Schuster, R.M., Fusar-Poli, P., \& Gonzalez, R. (2013). Effects of cannabis on neurocognitive functioning: recent advances, neurodevelopmental influences, and sex differences. Neuropsychology Review, 23, 117-137. doi: 10.1007/s11065012-9222-1

Crane, N.A., Schuster, R.M., \& Gonzalez, R. (2013). Preliminary evidence for a sex-specific relationship between amount of cannabis use and neurocognitive performance in young adult cannabis users. Journal of the International Neuropsychological Society : JINS, 19, 1009-1015. doi: 10.1017/S13556177130 0088X

Crane, N.A., Schuster, R.M., Mermelstein, R.J., \& Gonzalez, R. (2015). Neuropsychological sex differences associated with age of initiated use among young adult cannabis users. Journal of Clinical and Experimental Neuropsychology, 37, 389-401. doi: 10.1080/13803395.2015.1020770

Cyders M.A. \& Smith G.T. (2007), Mood-based rash action and its components: Positive and negative urgency. Personality and Individual Differences, 43, 839-850. doi: 10.1016/j.paid.2007. 02.008 .

Endicott, J. \& Spitzer, R.L. (1978). A diagnostic interview: The schedule for affective disorders and schizophrenia. Archives of General Psychiatry. 35, 837-843. doi: 10.1001/archpsyc.1978. 01770310043002

Ehrenreich, H., Rinn, T., Kunert, H.J., Moeller, M.R., Poser, W., Schilling, L., ... Hoehe, M.R. (1999). Specific attentional dysfunction in adults following early start of cannabis use. Psychopharmacology, 142, 295-301. doi: 10.1007/ s002130050892

Fletcher, P.C., \& Henson, R.N. (2001). Frontal lobes and human memory: Insights from functional neuroimaging. Brain, 124, 849-881. doi: 10.1093/brain/124.5.849

Fontes, M.A., Bolla, K.I., Cunha, P.J., Almeida, P.P., Jungerman, F., Laranjeira, R.R., ... Lacerda, A.L. (2011). Cannabis use before age 15 and subsequent executive functioning. The British Journal of Psychiatry, 198, 442-447. doi: 10.1192/bjp.bp.110.077479

First, M.B., Williams, J.B.W., Kargs, R.S., \& Spitzer, R.L. (2015). Structured clinical interview for DSM-5-research version (SCID-5 for DSM-5, research version; SCID-5-RV). Arlington, VA: American Psychiatric Association.

Giedd, J.N., Blumenthal, J., Jeffries, N.O., Castellanos, F.X., Liu, H., Zijdenbos, A., . . R Rapoport, J.L. (1999). Brain development during childhood and adolescence: A longitudinal MRI study. Nature Neuroscience, 2, 861-863. doi: 10.1038/13158

Gogtay, N., Giedd, N., Lusk, L., Hayashi, K.M., Greenstein, D., Vaituzis, A., ... Thompson, P.M. (2004). Dynamic mapping of human cortical development during childhood through early adulthood. Proceedings of the National Academy of Sciences, 101, 8174-8179. doi: 10.1073/pnas.0402680101

Gogtay, N., \& Thompson, P.M. (2010). Mapping gray matter development: implications for typical development and vulnerability to psychopathology. Brain and Cognition, 72, 6-15. doi: 10.1016/j. bandc.2009.08.009

Grant, J.E., Chamberlain, S.R., Schreiber, L., \& Odlaug, B.L. (2012). Neuropsychological deficits associated with cannabis 
use in young adults. Drug and Alcohol Dependence, 121, 159-162. doi: 10.1016/j.drugalcdep.2011.08.015

Gosling, S. D., Rentfrow, P. J., \& Swann, W. B., Jr. (2003). A very brief measure of the big five personality domains. Journal of Research in Personality, 37, 504-528. doi: 10.1016/S00926566(03)00046-1

Gur, R.C., Richard, J., Calkins, M.E., Chiavacci, R., Hansen, J.A., Bilker, W.B., ... Gur, R.E. (2012). Age group and sex differences in performance on a computerized neurocognitive battery in children age 8-21. Neuropsychology, 26, 251-265. doi: 10.1037/a0026712

Gur, R.C., Richard, J., Hughett, P., Calkins, M.E., Macy, L., Bilker, W.B., ... Gur, R.E. (2010). A cognitive neurosciencebased computerized battery for efficient measurement of individual differences: Standardization and initial construct validation. Journal of Neuroscience Methods, 187, 254-262. doi: 10.1016/ j.jneumeth.2009.11.017

Gruber, S.A., Sagar, K.A., Dahlgren, M.K., Racine, M., \& Lukas, S.E. (2012). Age of onset of marijuana use and executive function. Psychology of Addictive Behaviors: Journal of the Society of Psychologists in Addictive Behaviors, 26, 496-506. doi: 10.1037/a0026269

Hall, W. \& Degenhardt, L. (2009). Adverse health effects of nonmedical cannabis use. Lancet, 373, 1389-1391. doi: 10.1016/ S0140-6736(09)61037-0

Heng, L., Beverley, J.A., Steiner, H., \& Tseng, K.Y. (2011). Differential developmental trajectories for CB1 cannabinoid receptor expression in limbic/associative and sensorimotor cortical areas. Synapse (New York, N.Y.), 65, 278-286. doi: 10.1002/ syn. 20844

Herkenham, M., Lynn, A.B., Little, M.D., Johnson, M.R., Melvin, L.S., de Costa, B.R., \& Rice, K.C. (1990). Cannabinoid receptor localization in brain. Proceedings of the National Academy of Sciences of the United States of America, 87, 1932-1936. doi: 10.1073/pnas.87.5.1932

Herrmann, E.S., Weerts, E.M., \& Vandrey, R. (2015). Sex differences in cannabis withdrawal symptoms among treatment-seeking cannabis users. Experimental and Clinical Psychopharmacology, 23(6), 415-421. doi: 10.1037/ pha0000053

Hindocha, C., Freeman, T.P., \& Curran, H.V. (2017). Anatomy of a joint: Comparing self-reported and actual dose of cannabis and tobacco in a joint, and how these are influenced by controlled acute administration. Cannabis and Cannabinoid Research, 2(1), 217-223. doi: 10.1089/can.2017.0024

Huttenlocher, P.R. \& Dabholkar, A.S. (1997). Regional differences in synaptogenesis in human cerebral cortex. The Journal of Comparative Neurology, 387, 167-178. doi: 10.1002/(sici) 1096-9861(19971020)387:2<167:aid-cne1>3.0.co;2-Z

Johnston, L.D., Miech, R.A., O’Malley, P.M., Bachman, J.G., Schulenberg, J.E., Patrick, M.E. (2020). Monitoring the future national survey results on drug use 1975-2019: Overview, key findings on adolescent drug use. Ann Arbor: Institute for Social Research, University of Michigan.

King, G. R., Ernst, T., Deng, W., Stenger, A., Gonzales, R.M., Nakama, H., \& Chang, L. (2011). Altered brain activation during visuomotor integration in chronic active cannabis users: Relationship to cortisol levels. The Journal of Neuroscience : The Official Journal of the Society for Neuroscience, 31, 17923-17931. doi: 10.1523/JNEUROSCI.4148-11.2011

Kramer, J.H., Delis, D.C., \& Daniel, M.H. (1988). Sex differences in verbal learning. Journal of Clinical Psychology, 44, 907-915. doi: 10.1002/1097-4679(198811)44:6<907:AID-JCLP227044 $0610>3.0 . \mathrm{CO} ; 2-8$

Lisdahl, K.M. \& Price, J.S. (2012). Increased marijuana use and gender predict poorer cognitive functioning in adolescents and emerging adults. Journal of the International Neuropsychological Society : JINS, 18, 678-688. doi: 10.1017/ S1355617712000276

Lisdahl, K.M. \& Tapert, S. (2012). Chronic effects of heavy alcohol and marijuana use on the brain and cognition in adolescents and young adults. In H.R. White \& D.L. Rabiner (Eds.), Duke series in child development and public policy. College drinking and drug use (pp. 63-82). New York, NY: Guilford Press.

Mackie K. (2005). Distribution of cannabinoid receptors in the central and peripheral nervous system. Handbook of Experimental Pharmacology, 168, 299-325. doi: 10.1007/3540-26573-2_10

Martín-Santos, R., Fagundo, A.B., Crippa, J.A., Atakan, Z., Bhattacharyya, S., Allen, P., Fusar-Poli, P., ... McGuire, P. (2010). Neuroimaging in cannabis use: A systematic review of the literature. Psychological Medicine, 40, 383-398. doi: 10. 1017/S0033291709990729

McQueeny, T., Padula, C.B., Price, J., Medina, K.L., Logan, P., \& Tapert, S.F. (2011). Gender effects on amygdala morphometry in adolescent marijuana users. Behavioural Brain Research, 224, 128-134. doi: 10.1016/j.bbr.2011.05.031

Medina, K.L., McQueeny, T., Nagel, B.J., Hanson, K.L., Yang, T.T., \& Tapert, S.F. (2009). Prefrontal cortex morphometry in abstinent adolescent marijuana users: subtle gender effects. Addiction Biology, 14, 457-468. doi: 10.1111/j.1369-1600. 2009.00166.x

Meier, M.H., Caspi, A., Ambler, A., Harrington, H., Houts, R., Keefe, R.S.E., . . Moffitt, T.E. (2012). Persistent cannabis users show neuropsychological decline from childhood to midlife. Proceedings of the National Academy of the United States of American, 109, E2657-E2664. doi: 10.1073/pnas.1206820109

Narimatsu, S., Watanbe, K., Yamamoto, I., \& Yoshimura, H. (1991). Sex difference in the oxidative metabolism of $\Delta^{9}$-tetrahydrocannabinol in the rat. Biochemical Pharmacology, 41, 1187-1194. doi: 10.1016/0006-2952(91)90657-Q

Owen, A.M., Downes, J.J., Sahakian, B.J., Polkey, C.E., \& Robbins, T.W. (1990). Planning and spatial working memory following frontal lobe lesions in man. Neuropsychologia, 28, 1021-1034. doi: 10.1016/0028-3932(90)90137-d

Pardini, D., White, H.R., Xiong, S., Bechtold, J., Chung, T., Loeber, R., \& Hipwell, A. (2015). Unfazed or dazed and confused: Does early adolescent marijuana use cause sustained impairments in attention and academic functioning?. Journal of Abnormal Child Psychology, 43, 1203-1217. doi: 10.1007/s10802-015-0012-0

Pope, H. G., Jr., Jacobs, A., Mialet, J.-P., Yurgelun-Todd, D., \& Gruber, S. (1997). Evidence for a sex-specific residual effect of cannabis on visuospatial memory. Psychotherapy and Psychosomatics, 66, 179-184. doi: 10.1159/000289132

Pironti, V.A., Lai, M.C., Müller, U., Dodds, C.M., Suckling, J., Bullmore, E.T., \& Sahakian, B.J. (2014). Neuroanatomical abnormalities and cognitive impairments are shared by adults with attention-deficit/hyperactivity disorder and their unaffected first-degree relatives. Biological Psychiatry, 76, 639-647. doi: 10.1016/j.biopsych.2013.09.025

Reavis, R. \& Overman, W.H. (2001). Adult sex differences on a decision-making task previously shown to depend on the orbital prefrontal cortex. Behavioral Neuroscience, 115, 196-206. doi 10.1037/0735-7044.115.1.196 
Robbins, T.W., Semple, J., Kumar, R., Truman, M.I., Shorter, J., Ferraro, A., ... Mathews, K. (1997). Effects of scopolamine on delayed-matching-to-sample and paired associates tests of visual memory and learning in human subjects: Comparison with diazepam and implications for dementia. Psychopharmacology, 134, 95-106. doi: 10.1007/s002130050430

Robinson, S.M., Sobell, L.C., Sobell, M.B., \& Leo, G.I. (2014). Reliability of the timeline followback for cocaine, cannabis, and cigarette use. Psychology of Addictive Behaviors, 28, 154162. doi: 10.1037/a0030992

Sahakian, B.J., Morris, R.G., Evenden, J.L., Heald, A., Levy, R., Philpot, M., \& Robbins, T.W. (1988). A comparative study of visuospatial memory and learning in Alzheimer-type dementia and Parkinson's disease. Brain, 111, 695-718. doi: 10.1093/ brain/111.3.695

Saunders, J.B., Aasland, O.G., Babor, T.F., de la Fuente, J.R., \& Grant, M. (1993). Development of the Alcohol Use Disorders Identification Test (AUDIT): WHO collaborative project on early detection of persons with harmful alcohol consumption-II. Addiction (Abingdon, England), 88, 791-804. doi: 10.1111/j. 1360-0443.1993.tb02093.x

Scott, J.C., Slomiak, S.T., Jones, J.D., Rosen, A., Moore, T.M., \& Gur, R.C. (2018). Association of cannabis with cognitive functioning in adolescents and young adults: A systematic review and meta-analysis. JAMA Psychiatry, 75, 585-595. doi: 10. 1001/jamapsychiatry.2018.0335

Sheehan, D.V., Lecrubier, Y., Sheehan, K.H., Amorim, P., Janavs, J., Weiller, E., ... Dunbar, G.C. (1998). The Mini-International Neuropsychiatric Interview (M.I.N.I.): The development and validation of a structured diagnostic psychiatric interview for DSM-IV and ICD-10. The Journal of Clinical Psychiatry, 59(Suppl 20), 22-57.

Solowij, N., Stephens, R.S., Roffman, R.A., Babor, T., Kadden, R., Miller, M., ... Marijuana Treatment Project Research Group (2002). Cognitive functioning of long-term heavy cannabis users seeking treatment. JAMA, 287, 1123-1131. doi: 10.1001/jama. 287.9.1123

Sowell, E.R, Thompson, P.M, Holmes, C.J., Jernigan, T.L., \& Toga, A.W. (1999). In vivo evidence for post-adolescent brain maturation in frontal and striatal regions. Nature Neuroscience, 2, 859-861. doi: 10.1038/13154

Sowell, E.R., Thompson, P.M., Tessner, K.D., \& Toga, A.W. (2001). Mapping continued brain growth and gray matter density reduction in dorsal frontal cortex: Inverse relationships during postadolescent brain maturation. The Journal of Neuroscience: The Official Journal of the Society for Neuroscience, 21, 88198829. doi: 10.1523/JNEUROSCI.21-22-08819.2001

Substance Abuse and Mental Health Services Administration. (2019). Key substance use and mental health indicators in the United States: Results from the 2018 national survey on drug use and health (HHS Publication No. PEP19-5068, NSDUH Series H-54). Rockville, MD: Center for Behavioral Health Statistics and Quality, Substance Abuse and Mental Health Services Administration.

Schuster, R.M., Hoeppner, S.S., Evins, A.E., \& Gilman, J.M. (2016). Early onset marijuana use is associated with learning inefficiencies. Neuropsychology, 30, 405-415. doi: 10.1037/ neu0000281

Schuster, R.M., Gilman, J., Schoenfeld, D., Evenden, J., Hareli, M., Ulysse, C., ... Evins, A.E. (2018). One month of cannabis abstinence in adolescents and young adults is associated with improved memory. The Journal of Clinical Psychiatry, 79, 17m11977. doi: 10.4088/JCP.17m11977

Van Laere, K., Koole, M., Sanabria Bohorquez, S.M., Goffin, K., Guenther, I., Belanger, M.G., ... Burns, H.D. (2008). Wholebody biodistribution and radiation dosimetry of the human cannabinoid type-1 receptor ligand 18F-MK-9470 in healthy subjects. Journal of Nuclear Medicine: Official Publication, Society of Nuclear Medicine, 49, 439-445. doi: 10.2967/jnumed.107. 047290

Wallace, A., Wade, N., \& Lisdahl, K. (2020). Impact of 2 weeks of monitored abstinence on cognition in adolescent and young adult cannabis users. Journal of the International Neuropsychological Society, 26(8), 776-784. doi: 10.1017/S1355617720000260

Watson, D., Weber, K., Assenheimer, J.S., Clark, L.A., Strauss, M.E., \& McCormick, R.A. (1995). Testing a tripartite model: I. Evaluating the convergent and discriminant validity of anxiety and depression symptom scales. Journal of Abnormal Psychology, 104, 3-14. doi: 10.1037//0021-843x.104.1.3

Wechsler, D. (1999). Wechsler Abbreviated Scale of Intelligence. New York, NY: The Psychological Corporation, Harcourt Brace \& Company.

Wechsler, D. (2001). Wechsler Test of Adult Reading. San Antonio, TX; Pearson.

Wetherington C.L. (2007). Sex-gender differences in drug abuse: A shift in the burden of proof?. Experimental and Clinical Psychopharmacology, 15, 411-417. doi: 10.1037/1064-1297. 15.5.411 